\title{
Cell Sleeping for Energy Efficiency in Cellular Networks: Is it Viable?
}

\author{
Xin Wang, Prashant Krishnamurthy, and David Tipper \\ Graduate Telecommunications and Networking Program \\ University of Pittsburgh, Pittsburgh, PA 15260 \\ \{xiw54, prashk, dtipper\}@ pitt.edu
}

\begin{abstract}
An approach advocated in the recent literature for reducing energy consumption in cellular networks is to put base stations to sleep when traffic loads are low. However, several practical considerations are ignored in these studies. In this paper, we aim to raise questions on the feasibility and benefits of base station sleeping. Specifically we analyze the interference and capacity of a coverage-based energy reduction system in CDMA based cellular networks using a simple analytical model and show that sleeping may not be a feasible solution to reduce energy consumption in many scenarios.
\end{abstract}

\section{INTRODUCTION}

Current cellular networks essentially operate in a $24 \times 7$ "always-on" mode. This is motivated by several factors such as the desire to provide highly available quality network performance to customers and to meet government regulatory requirements (e.g., E-911 localization mandate). Cellular networks are designed such that the capacity allocated to a specific cell site is determined to meet peak busy period (e.g., hour) traffic load quality of service requirements (e.g., $2 \%$ call blocking [6]). However, the traffic load experiences significant variations with time of year, time of day, geographic location, weather and other societal factors [7]. Note that, cellular networks and base stations in particular consume significant power even when not carrying traffic. This has led to recognition of the need for energy efficiency in cellular networks by industry, standards groups, and the research community [1]-[4].

Within the cellular network, base stations (BSs) are responsible for most of the energy consumption, consuming 70-95\% or more of the network power depending on the network topology, BS configuration, radio technology and data rate used. An intuitive approach suggested in the literature [2][5] for reducing energy consumption in cellular networks is to put base stations to sleep when their load is low. We will refer to such schemes as coverage-based energy reduction management of cellular networks. However, the work in this area ignores some practical realities that we start to investigate in this paper. For instance, if a cell is put in a sleep mode and one wants to avoid coverage holes, it is not clear how much extra power is needed from neighboring cells to cover the area previously serviced by the sleeping cell. Further, the increased power of neighbor cells may result in increased interference or capacity reduction elsewhere.

In this paper, we study the feasibility and benefits of "base station sleeping" for energy conservation. We quantitatively evaluate the power-savings and analyze the interference and capacity under "base station sleeping" in CDMA cellular networks using a simple analytical model. We consider two scenarios to examine the benefits or drawbacks of coveragebased energy reduction. In the first scenario, we assume that a base station covering a central cell in a hexagonal grid is put to sleep while the other base stations work in the normal mode without any increase in transmit power. We then analyze the impact on both the reverse-link and the forward-link of the surrounding cells. The numerical results show that the natural extension of coverage from neighboring cells into the sleeping cell is limited. Further, the coverage extension reduces with the number of users since an acceptable $E_{b} / I_{0}$ cannot be achieved. Even when a cell is put to sleep with a very low traffic load in the system, neighboring cells may still not be able to cover the slept area without holes in the coverage. This result implies that the transmit power of neighboring cells must be increased to ensure there are no coverage holes. In the second scenario, while sleeping the base station in a cell at the center of the grid, the transmit powers of only two neighboring cells are changed (identically) to guarantee the minimum received $E_{b} / I_{0}$ for mobile users in the coverage hole. We evaluate the capacity in the second scenario and show that the overall capacity of neighbor cells is reduced due to the two extended cells with increased power. Further we discuss whether it is worth using base station sleeping schemes and point out a number of business and regulatory issues in addition to the concerns raised by our analysis.

The rest of the paper is organized as follows. In Section 2, we present a brief overview of related work. Section 3 presents a simple signal-to-interference ratio model for the reverse-link and forward-links of a CDMA system and the two scenarios for base station sleeping. Section 4 presents the evaluation of the two scenarios. We discuss the merits of sleeping in Section 5 and conclude in Section 6.

\section{RELATED WORK}

Saving energy by carefully switching off base stations with low traffic has been suggested in [1]-[5]. This approach is sometimes called as turning to the sleeping mode. When some cells are switched off, the radio coverage and service provisioning for those areas will have to be taken care of by neighboring cells. A second approach is to adaptively adjust the cell size according to the temporal fluctuation of the traffic load [8]-[10]. This time-variant operation is called cell breathing or cell zooming. At the receiver, in order to maintain a specific signal-to-interference ratio, the transmitter 
either increases the transmit power or decreases the distance at which a receiver can be when the noise floor (which is proportional to the number of users) becomes higher. In other words, a BS's coverage shrinks when the traffic load grows and grows when the traffic load drops.

Both approaches aim at energy savings that exploit the variation of the traffic load by dynamically adjusting the network topology and coverage. Such previously proposed strategies of this "coverage-based energy reduction" only focus on the traffic load analysis and dynamic network topology designs but ignore wireless link factors that must be accounted for in a practical cellular environment. In most of the papers, a simplifying assumption is made that the neighbor cells which take over the load of the sleeping cells do not have to increase their transmit power (or this increase is negligible) [3], [4], [8]. Thus the obvious conclusion is that the network saves energy when some base stations are turned off or when some cells are zooming in because the transmit powers of neighbors remain unchanged. The authors in [9] simulated a centralized algorithm and a distributed algorithm based on a Base StationMobile User matrix to control cell zooming. However they did not consider the geographic location of each base station and each mobile user or the the link level power budget.

Here we ask the question whether a base station needs to increase its transmit power when it has to provide coverage to support the mobile users in its neighboring cell and if so by how much. This could be to mitigate degradation due to path loss or to maintain a specific signal-to-interference ratio. Finally, in CDMA networks, the capacity of a cell is interference limited. If the transmit power of one base station increases, the interference in other cells in the same network will be affected which could possibly cause capacity degradation. Therefore, without considering all these factors in cellular networks, it is not possible to claim that "base station sleeping" is efficient or feasible.

\section{AnAlytical Model And Scenarios}

We investigate using a simple model whether or not it is possible to obtain power savings by sleeping cells yet maintaining full coverage. Consider a CDMA based network (e.g., UMTS, cdma2000) with $J$ homogenous cells arranged in a hexagonal grid such that each cell has six neighbors as shown in Figure 1. Let the initial radius of each of cell be $R$ and $P_{t}$ denote the initial transmit power of each cell. We assume that the users are uniformly distributed everywhere in the grid and the number of users in each cell is initially $N+1$. Let us suppose that at some later time (e.g., night) a central area (cell 0 ) has a much smaller load (only $M \ll N+1$ users in its coverage). Also two of its neighbors (cells 1 and 2) have a slightly smaller load (say $K_{1}, K_{2}>M$ but $K_{1}, K_{2}<N+1$ users) in the same time period. Since $M \ll N+1$, it may be possible to sleep cell 0 to reduce energy consumption. Thus, the Radio Network Controller (RNC) directs cell 0 to sleep. The $M$ mobile stations in cell 0 are handed over to cells 1 and 2 ( $M_{1}$ users to cell 1 and $M_{2}$ users to cell 2 respectively). We make the simplifying assumption that $N+1=M_{1}+K_{1}=M_{2}+K_{2}$ so that there are $N+1$ users at all times in each cell. We will consider 2 scenarios in this paper. In the first scenario, the other cells operate normally as

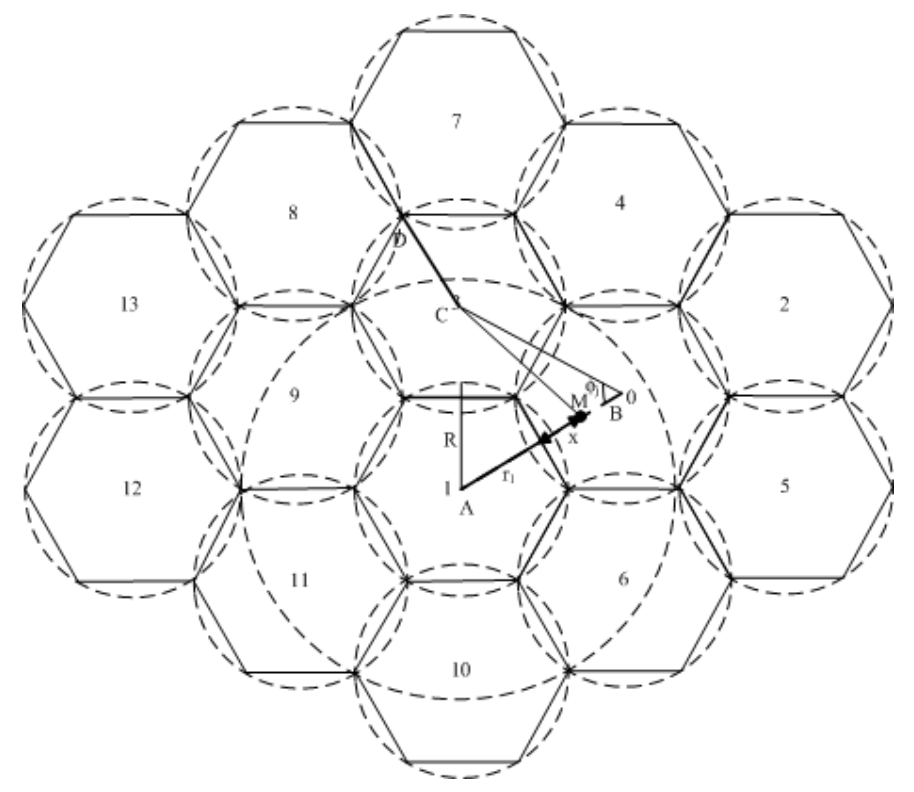

Fig. 1. Cellular Topology for CDMA sleeping scenarios

before, i.e., the RNC only sleeps cell 0 and leaves the coverage of cell 0 geographic space to the natural extension of coverage in cells 1 and 2 that occurs due to the smaller interference. We will see that this typically results in coverage holes. In the second scenario, the RNC directs the base stations in cells 1 and 2 (BS $1 \&$ BS 2) to increase their transmit powers to ensure that there is no coverage hole in cell 0's original coverage area. In order to analyze the two scenarios we first consider the SIR and capacity analysis of the system.

Mathematical Models for $E_{b} / I_{0}$ and Capacity: We consider one carrier in a CDMA cellular system. To maintain a call's quality, each signal arrives at its intended receiver with the minimum required signal-to-interference ratio (SIR or $E_{b} / I_{0}$ ).

1) Reverse-link SIR: An upper bound on the capacity of the reverse-link occurs because mobile stations eventually have insufficient power to achieve the required $E_{b} / I_{0}$ at the base station. Within a cell, power control is employed to ensure that the signal strength $S$ of every signal received at the base station is approximately the same. For any mobile station, the in-cell interference is then $N S$, where $N+1$ is the number of mobile stations within the cell. This term is the primary source of interference on the reverse-link. The interference from mobile stations outside the cell is the secondary source of interference and can be taken to be a fraction $\beta$ of the incell interference. For large values of $N$, all interference can be reduced by a factor $v$ which reflects the mean voice activity across all active channels on the reverse-link. As shown in [6] this leads to :

$$
\frac{E_{b}}{I_{0}}=\frac{G S}{\text { Noise }+v(1+\beta) N S}
$$

where $G$ is the processing gain, $S$ is the received signal strength, $v$ is the voice activity factor, $\beta$ is the interference factor from other cells, Noise is the thermal noise, and $N$ is the number of interfering mobile stations in the cell. 
2) Forward-link SIR: The forward-link capacity is limited by $E_{b} / I_{0}$ when mobile stations are located at the cell boundary [11]. Consider cell 1 in Figure 1 . The $E_{b} / I_{0}$ on the forwardlink can be expressed as

$$
\frac{E_{b}}{I_{0}}=\frac{G S}{N o i s e+\beta \sum_{j=2}^{J-1} \sum_{i=0}^{N} r_{j}^{-\alpha} v P_{t}+\sum_{i=1}^{N} r_{1}^{-\alpha} v P_{t}}
$$

where $J$ is the number of cells considered and $r_{j}^{-\alpha} P_{t}$ is the received power at a tagged mobile a distance $r_{j}$ from BS $j$. The received signal strength at the edge of cell 1 is given by $S=r_{1}^{-\alpha} P_{t}$, where the path-loss exponent is $\alpha$. Power control is not included in this model and neither is the shadow fading. The second term in the denominator is the inter-cell interference and the last term is the intra-cell interference. Note, that when one cell is assumed to be sleeping, the number of interfering cells will be $J-2$. Simplifying,

$$
\begin{aligned}
\frac{E_{b}}{I_{0}}= & \frac{G}{\frac{1}{S N R}+\beta \sum_{j=2}^{J-1} \sum_{i=0}^{N} v\left(\frac{r_{j}}{r_{1}}\right)^{-\alpha}+\sum_{i=1}^{N} v} \\
= & \frac{G}{\frac{1}{S N R}+v(N+1) \beta \sum_{j=2}^{J-1}\left(\frac{r_{j}}{r_{1}}\right)^{-\alpha}+v N}
\end{aligned}
$$

3) Capacity on the Forward Link: According to Eq.(19) in [11], the capacity can be determined by the limits on the radiated power and by the $E_{b} / I_{0}$ requirement at the mobile receiver. However, unlike the reverse-link, the inter-cell interference is typically the primary source of interference. The capacity will thus depend on the mobile's position with respect to its serving cell as well as its neighbor cells. Shadow fading is also taken into account and the capacity on the forward link is derived in [11]. It is given by:

$$
N=\frac{1+\frac{G}{v}\left(\frac{I_{0}}{E_{b}}-\frac{1}{S N R}\right)}{1+\beta \sum_{j=2}^{J-1}\left(\frac{r_{j}}{r_{1}}\right)^{-\alpha} E\left[\Phi_{j}\left(\zeta_{j}, \frac{r_{1}}{r_{j}}\right) 10^{\zeta_{j} / 10}\right]}-1
$$

where

$$
\begin{aligned}
& E\left[\Phi_{j}\left(\zeta_{j}, \frac{r_{1}}{r_{j}}\right) 10^{\zeta_{j} / 10}\right]=\exp \left(\frac{\sigma \ln (10)}{10}\right)^{2} \\
& \left\{1-Q\left[\frac{10 \alpha \log \left(\frac{r_{j}}{r_{1}}\right)}{\sqrt{2} \sigma}-\frac{\sqrt{2} \ln (10) \sigma}{10}\right]\right\}
\end{aligned}
$$

Here $\sqrt{2} \sigma$ is the standard deviation of $\zeta_{j}$, a normally distributed random variable that corresponds to the shadow fading associated with the $j$-th base station and $Q$ is the tail probability of a standard normal random variable.

\section{Evaluation}

In this section, we employ the analytical expressions presented above to evaluate the practicality of the two dimming scenarios. For the first scenario, we (a) analyze the impact on the reverse-link and the forward-link and (b) determine if the natural coverage extension of cells is sufficient to overcome the hole created by a cell sleeping. For the second scenario, we (a) calculate how much extra power is needed to cover the hole created by the sleeping cell and (b) determine the capacity changes before and after sleeping and with or without the extra transmit power. We evaluate both scenarios using parameters typical for a UMTS network [12], specifically, for $12.2 \mathrm{Kbps}$ voice traffic from a mobile user with speed $120 \mathrm{~km} / \mathrm{h}$ the processing gain is $G=25 \mathrm{~dB}$ and the required $E_{b} / I_{0}$ $=5 \mathrm{~dB}$. Similarly, for $144 \mathrm{Kbps}$ real time data traffic the processing gain is $G=14.3 \mathrm{~dB}$ and the required $E_{b} / I_{0}=1.5$ $\mathrm{dB}$. Here we consider two voice activity factor values $v=\frac{3}{8}$ and $v=\frac{1}{2}(v=1$ for data) and two propagation coefficient values $\alpha=2$ (corresponding to free space) and $\alpha=4$. We assume that the thermal noise $-169.0 \mathrm{dBm}$ [12] is negligible compared to the other interference components.

\section{A. Scenario 1: No Increase in Transmit Power}

1) Impact on the Reverse-link: On the reverse-link, from Eq. 1, the number $N$ of interfering mobile stations (MSs) in a cell has to be less than a specific threshold to maintain the $\left(E_{b} / I_{0}\right)$ ratio level. In our assumption, the total number of MSs in cell 1's extended coverage will not exceed the number in cell 1's original coverage. Therefore, the interference will not increase after handover of the users in the sleeping cell. The required received power at Base Station 1 from the MSs in cell 0 will not change. However, the distances from the mobile stations in cell 0 to Base Station 1 are larger than what they were to Base Station 0. Assuming path loss $\propto$ distance $^{-\alpha}$, we know that to maintain the minimum required $\left(E_{b} / I_{0}\right)$ at Base Station 1, mobile stations originally in cell 0 must increase their transmit power. In other words, the neighbor cells may be able to support sleeping by handling the mobile stations, but with the mobile stations expending more energy. We don't consider this situation here since most of the energy consumption is at the cell sites.

2) Impact on the Forward-link: On the forward-link, by sleeping cell 0 , a significant interference component in cells 1 and 2 has been removed and intuitively, there must be some coverage extension. We assume that the farthest mobile station that Base Station 1 can support is ' $\mathrm{M}$ ' along line segment $\overline{A B}$ in Figure 1. We can see that $\mathrm{M}$ is at a distance of $r_{j}=$ $\sqrt{D_{j}^{2}+r_{1}^{2}-2 D_{j} r_{1} \cos \left(\phi_{j}\right)}$ from the $j$ th neighboring Base Station as shown in Figure 1 where $D_{j}$ is the distance between the center of cell 1 and the $j$ th Base Station and $r_{1}$ is the distance of $\mathrm{M}$ from base station 1. For this case, Eq. 3 reduces to:

$$
\frac{E_{b}}{I_{0}}=\frac{G}{\beta v(N+1) \sum_{j=2}^{J-1}\left(\frac{r_{j}}{r_{1}}\right)^{-\alpha}+v N}
$$

We are interested in calculating the coverage extension $x$, the distance between $\mathrm{M}$ and the edge of cell 1 , also along $\overline{A B}$. Let $x=r_{1}-R$, where $R$ is the original cell radius, $x$ can be calculated by solving Eq. 5 (with $x$ included in $r_{1}$ and $r_{j}$ ) for different values of the number of users $N$ per cell. Figure 2 shows $x$ normalized to the original cell radius $R$ when we consider all the interfering cells in Figure 1. We 
see that $x$ will decrease as the load increases. Similarly, $x$ decreases as $v$ increases representing more active users for the case of voice traffic. Interestingly, $x$ decreases as $\alpha$ decreases. This can be explained as follows when $\alpha=2$ the signals from Base Station 1 propagate farther, but the interference from other cells also increases greatly resulting in an overall reduction in the effective range of Cell 1 . Note for the data traffic case $(v=1)$ only a small number of users can be supported for any cell extension $(x>0)$. Observe that for all the cases considered, $x<1$ implying that a coverage hole exists. This means the transmit power of Base Station 1 must be increased if the neighboring cell is assigned to cover part of the sleeping area. Notice that if shadow fading is taken into account, a further increase in transmit power may be needed.
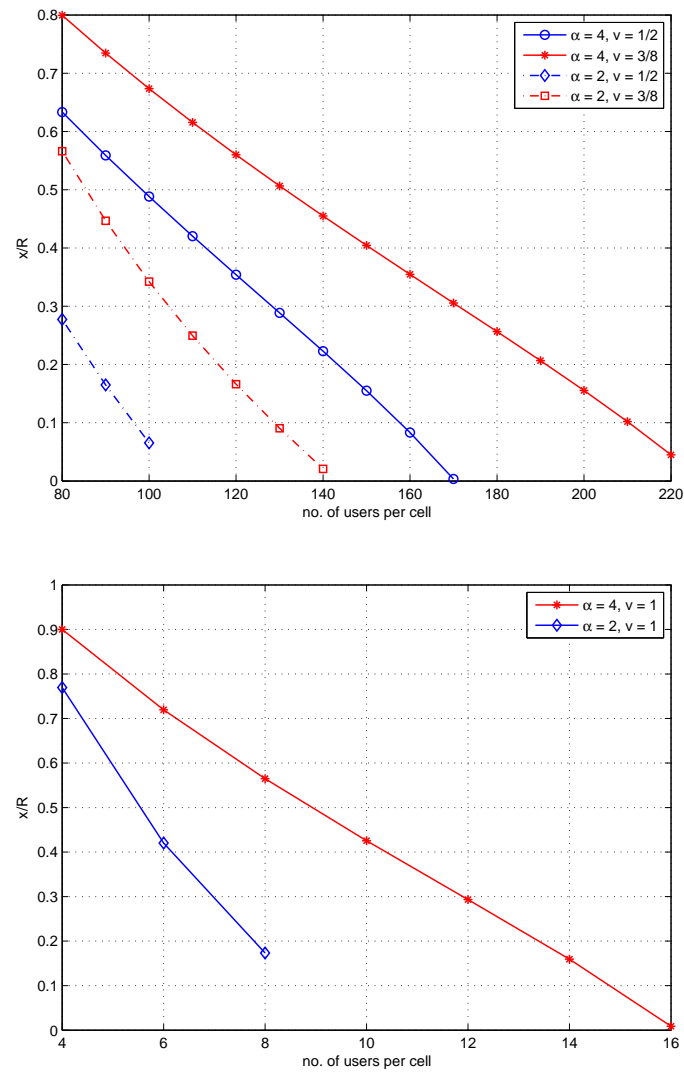

Fig. 2. Extended coverage versus number of users for voice (top) and data (bottom)

The same conclusion is demonstrated by a capacity analysis on the forward-link using Equation 4 which takes shadow fading into account (we used $\sigma=4$ in the numerical results). The capacity can be calculated along line segment $\overline{A B}$ from Base Station 0 to Base Station 1. We first calculate the capacity when Cell 0 is awake and then when Cell 0 is sleeping. In both cases, the capacity curve approaches something like a capacity limit when $x=0.6 R(r=1.6 R$ where $r=x+R)$ as shown in the Figure 3 (for example, with voice traffic this is $\approx 100$ users with $\alpha=4, v=1 / 2$ and $\approx 155$ users when $v=3 / 8$ ). Observe that the number of data users supported is much less than voice only traffic. In either traffic case, without increasing the transmit power, the neighbor cells cannot cover the hole created by the sleeping cell.
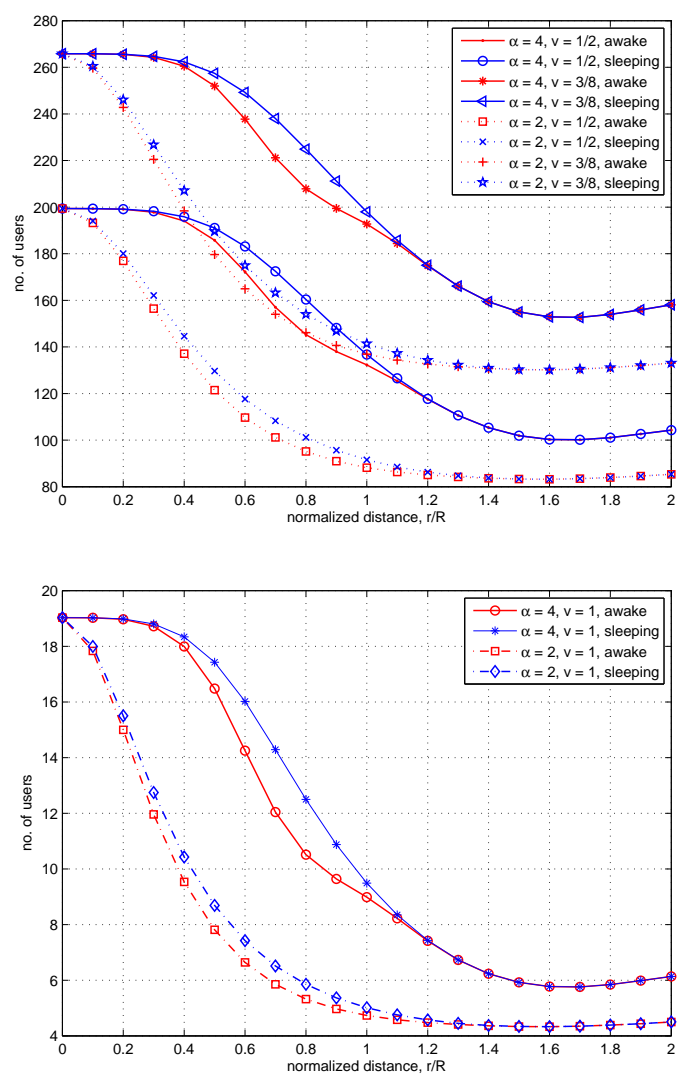

Fig. 3. Capacity on the Forward-link for Voice (top) and Data (bottom)

\section{B. Scenario 2: Two Neighbor Cells Expand Coverage}

In the last section, we saw that if the area of cell 0 is equally divided and each half is taken care of by cell 1 and 2 , the transmit power of base station 1 and 2 must increase. Otherwise, the system will be left with coverage holes. We look at the following questions next: (a) By how much does the transmit power need to increase? (b) What is the impact of this increase on the interference in the CDMA system?

1) Increase in Transmit Power: From Eq. 5, the required transmit power at the BS can be derived by setting $x$ to be equal to $R$. We get:

$$
\frac{P_{1}}{P_{0}}=\frac{\beta v(N+1) \sum_{j=3}^{J-1}\left(\frac{r_{j}}{r_{1}}\right)^{-\alpha}}{\frac{G I_{0}}{E_{b}}-v N-\beta v(N+1)\left(\frac{r_{2}}{r_{1}}\right)^{-\alpha}}
$$

The normalized required transmit power from this solution is shown in Figure 4. It is apparent that BSs that expand coverage need significant increases in the amount of transmit power even when the load is not high (e.g., the increased power $P_{1}$ is larger than the original power $P_{0}$ by $3 \mathrm{~dB}$ or two times when $N=4$ users with $\alpha=4, v=1$ with data). 

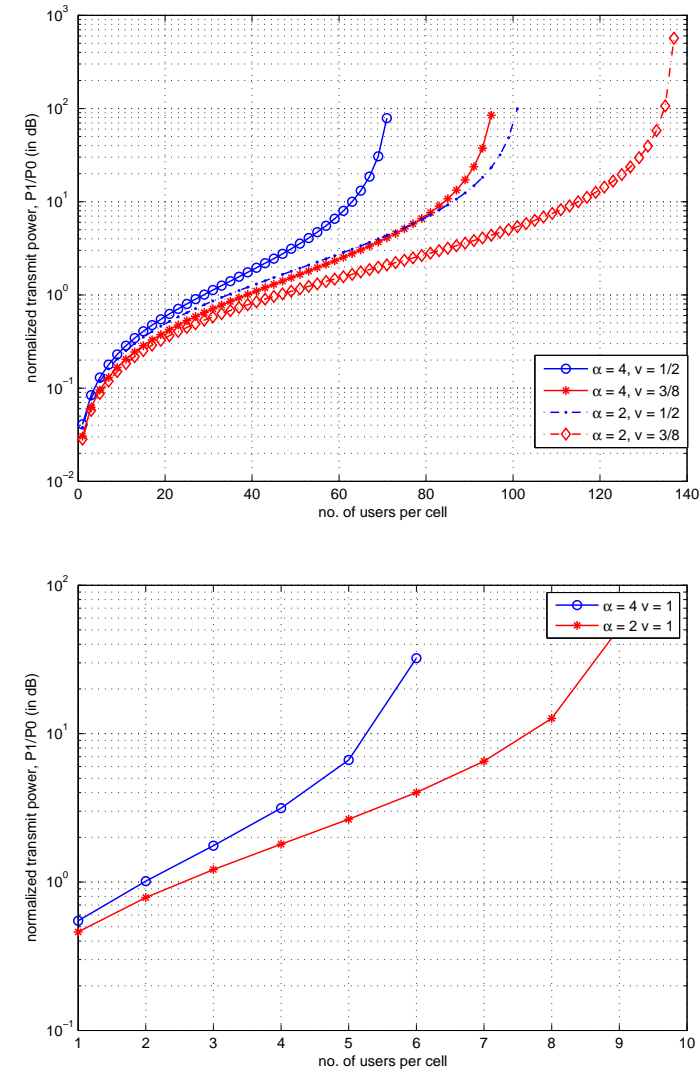

Fig. 4. Required Transmit Power of the Neighbor Cells for Voice (top) and Data (bottom)

2) Capacity under Increased Interference: Next, the same methodology and consideration used in [11] is adopted here to determine the capacity along line $\overline{C D}$ in cell 3 , a major victim of cell 1 and 2's extension of coverage. All the interfering cells of cell 3 are shown in Figure 1. The received signal power is $S=r_{j}^{-\alpha} P_{0}$ for the user from the $j$ th cell. In the extended cell, the signal power is $S=r_{j}^{-\alpha} P_{1}$. To use a reasonable value of $P_{1}$, we let the minimum received power at the cell edge be $P_{r}$ both before and after cell expansion. Assuming a simple path-loss model, $P_{r}=P_{0} \times K \times R^{-\alpha}=P_{1} \times K \times(2 R)^{-\alpha}$ which implies that $P_{1}=2^{\alpha} P_{0}$. The external cell SIR [11] is

$$
\begin{aligned}
I_{e x t} & =\frac{\beta}{G}\left(\sum_{j=4}^{J-1} \sum_{i=0}^{N} v_{i j}\left(\frac{r_{j}}{r_{1}}\right)^{-\alpha} 10^{\zeta_{j} / 10}\right. \\
& \left.+2^{\alpha} \sum_{j=1}^{2} \sum_{i=0}^{N} v_{i j}\left(\frac{r_{j}}{r_{1}}\right)^{-\alpha} 10^{\zeta_{j} / 10}\right) \\
& =\frac{\beta v(N+1)}{G}\left(\sum_{j=4}^{J-1}\left(\frac{r_{j}}{r_{1}}\right)^{-\alpha} 10^{\zeta_{j} / 10}\right. \\
& \left.+2^{\alpha} \sum_{j=1}^{2}\left(\frac{r_{j}}{r_{1}}\right)^{-\alpha} 10^{\zeta_{j} / 10}\right)
\end{aligned}
$$

and the required SIR is

$$
E\left[\frac{E_{b}}{I_{0}}\right] \geqslant \frac{1}{\frac{v N}{G}+\frac{\beta v(N+1) F}{G}+\frac{\beta v(N+1) Q}{G}+\frac{1}{S N R}}
$$

where

$$
F=\sum_{j=4}^{J-1}\left(\frac{r_{j}}{r_{1}}\right)^{-\alpha} E\left[\Phi_{j}\left(\zeta_{j}, \frac{r_{1}}{r_{j}}\right) 10^{\zeta_{j} / 10}\right]
$$

and

$$
Q=2^{\alpha} \sum_{j=1}^{2}\left(\frac{r_{j}}{r_{1}}\right)^{-\alpha} E\left[\Phi_{j}\left(\zeta_{j}, \frac{r_{1}}{r_{j}}\right) 10^{\zeta_{j} / 10}\right]
$$

So that, we get

$$
N=\frac{\left(\frac{I_{0}}{E_{b}}-\frac{1}{S N R}\right) \frac{G}{v}+1}{1+\frac{1}{2}(F+Q)}-1
$$
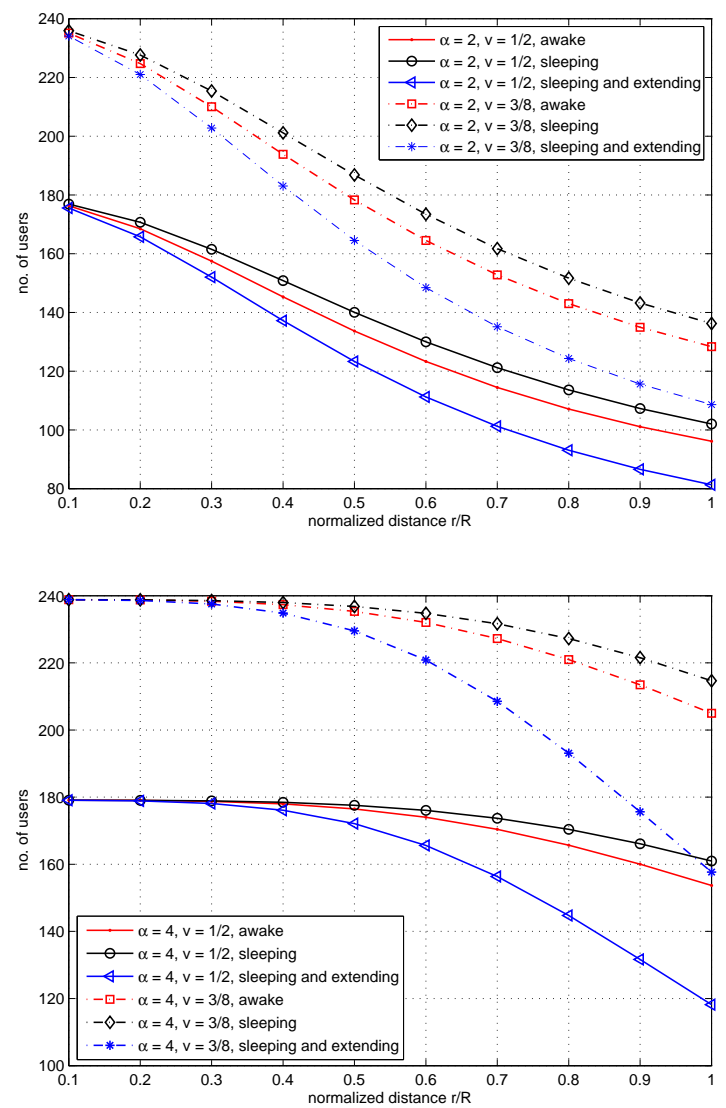

Fig. 5. Forward-link capacity as a function of the distance along line CD for voice - top $(\alpha=2)$ and bottom $(\alpha=4)$

The capacity curves are shown in Figures 5 and 6 for voice and data. In both cases the capacity of the neighbor cell has a slight improvement after a cell is slept, without expanding any cells to make up the coverage. However, the capacity will suffer significantly if some cells extend their radius to cover the central area. Since we assumed that all of the cells have the same number of users $N+1$, in it appears that sleeping will actually work only if the overall load in all of the neighboring 

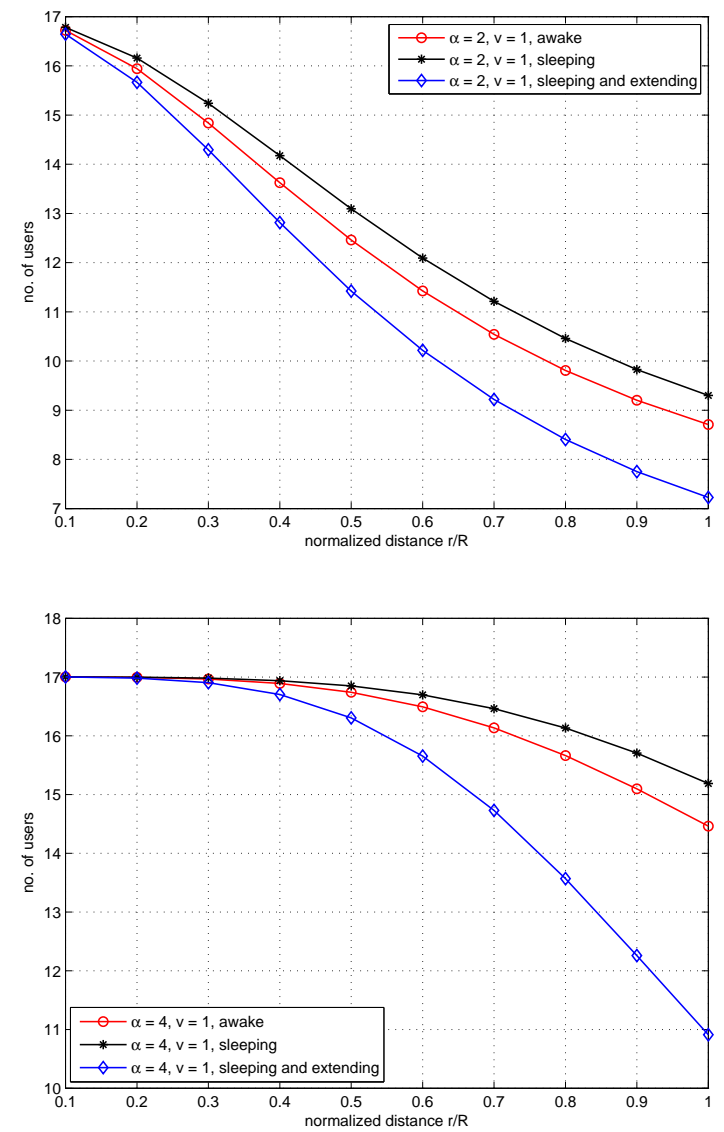

Fig. 6. Forward-link capacity as a function of the distance along line CD for data - top $(\alpha=2)$ and bottom $(\alpha=4)$

cells is small. Thus it is not sufficient that the load drops in the geographical area corresponding to the cell that is slept.

\section{DisCusSION}

The base station sleep model studied in this paper has been adopted in [2]-[5]. Our analysis seems to indicate that sleeping cells is not something that can be done without substantial planning and management to account for load and cell sizes. Similar results can also be obtained from some other scenarios which are not listed due to space limit. For instance, identically change the transmit powers of six neighboring cells instead of only two to guarantee the coverage. In all the scenarios we did not consider specific cell sizes, heterogeneous cells (cells of varying sizes), or a variety of path-loss models, but even the simplified analyses makes it clear that naive assumptions of simply allowing neighboring base stations to cover the area previously covered by a sleeping cell are not sufficient. Further, the amount of power savings from sleeping may not be large enough. For example, if we assume that most of the energy consumption is from the transmit power, the initial total power (before sleeping) is $14 P_{0}$ and the power consumption after sleeping is $P_{u}=11 P_{0}+2 P_{1}$. Thus, for power savings, $P_{u}<14 P_{0}$ or $2^{\alpha}<1.5$ or $\alpha<0.585$ which is not a realistic possibility. Of course, additional energy is saved due to the power down of other components in a base station. We also note here that sleeping cells may not be viable for operational reasons as well. Current cellular networks essentially operate in a $24 \times 7$ always on mode. This is due to several factors such as the need of some cellular technology to maintain synchronization (e.g., cdma2000) of base stations, inability to rapidly power down/up equipment, and E-911 localization requirements.

\section{CONCLUSION}

In this paper, we examine whether sleeping cell-sites can naturally allow neighboring cell sites to cover the geographical area serviced by the sleeping cell. Using fairly simple models for capacity and SIR, we show that the problem is not as inconsequential as previously assumed and that sleeping cell sites may not necessarily be viable or energy efficient. The load in the entire geographical area, not simply the area where the load is low needs to be taken into account to suitably sleep cells making this a more difficult network management problem than previously assumed. Using models and simulations that accurately characterize load and interference across a wider geographical area is necessary to determine when base station sleeping is feasible and when it is not.

\section{ACKNOWLEDGMENT}

This research work was supported in part by a seed grant from the University of Pittsburgh Center for Energy.

\section{REFERENCES}

[1] Eunsung Oh, Bhaskar Krishnamachari, Xin Liu, Zhisheng Niu, Toward Dynamic Energy-Efficient Operation of Cellular Network Infrastructure, IEEE Communications Magazin, June 2011.

[2] Remco Litjens and Ljupco Jorguseski, Potential of Energy-Oriented Network Optimisation: Switching Off Over-Capacity in Off-Peak Hour, IEEE 21st International Symposium on Personal Indoor and Mobile Radio Communications (PIMRC), Sept. 2010.

[3] MA Marsan, L. Chiaraviglio, D. Ciullo, and M. Meo., Optimal energy savings in cellular access networks, Proc. IEEE ICC'09 Workshop, GreenComm, June 2009.

[4] Jie Gong, Sheng Zhou, Zhisheng Niu and Peng Yang, Traffic-aware base station sleeping in dense cellular networks, 18th International Workshop on Quality of Service (IWQoS), June 2010.

[5] Oliver Blume, Harald Eckhardt, Siegfried Klein, Edgar Kuehn, and Wieslawa M. Wajda, Energy Savings in Mobile Networks Based on Adaptation to Traffic Statistics, Bell Lab Technical Journal Vol.15, No. 2, 77-94, 2010.

[6] Kyoung Il Kim, Handbook of CDMA System Design, Engineering, and Optimization, USA, 2000.

[7] D. Willkomm et al, Primary User Behavior in Cellular Networks and Implications for Dynamic Spectrum Access, IEEE Commun. Mag., vol.47, no.3, Mar.2009, pp.88-95.

[8] S. Bhaumik, G. Narlikar, S. Chattopadhyay, and S. Kanugovi, Breathe to Stay Cool: Adjusting Cell Sizes to Reduce Energy Consumption, Proc. 1st ACM SIGCOMM Workshop on Green Networking (GreenNetworking '10) (New Delhi, Ind., 2010), pp. 41-46.

[9] Z. Niu, Y. Wu, J. Gong, and Z. Yang, Cell Zooming for Cost-Efficient Green Cellular Networks, IEEE Communication Magazine, Nov.2010.

[10] D. Tipper, A. Rezgui, P. Krishnamurthy, and P. Pacharintanakul, Dimming Cellular Networks, Proc. IEEE Globecom, Dec., 2010.

[11] C. Lee, R. Steele, Effect of Soft and Softer Handoffs on CDMA System capacity, IEEE Transactions on Vehicular Technology, Aug. 1998, pp. 830-841.

[12] Chris Braithwaite and Mike Scott, UMTS Network Planning and Development: Design and Implementation of the $3 G$ CDMA Infrastructure, UK, 2003. 